\title{
Integrated Make-or-Buy and Facility Layout Problem with Multiple Products
}

DOI:

10.1007/978-3-319-20863-3_9

\section{Document Version}

Accepted author manuscript

Link to publication record in Manchester Research Explorer

\section{Citation for published version (APA):}

Hillebrand, B., \& Pishchulov, G. (2016). Integrated Make-or-Buy and Facility Layout Problem with Multiple Products. In D. Mattfeld, T. Spengler, J. Brinkmann, \& M. Grunewald (Eds.), Logistics Management: Contributions of the Section Logistics of the German Academic Association for Business Research, 2015, Braunschweig, Germany (pp. 121-132). (Lecture Notes in Logistics). Springer Nature. https://doi.org/10.1007/978-3-319-208633_9

\section{Published in:}

Logistics Management

\section{Citing this paper}

Please note that where the full-text provided on Manchester Research Explorer is the Author Accepted Manuscript or Proof version this may differ from the final Published version. If citing, it is advised that you check and use the publisher's definitive version.

\section{General rights}

Copyright and moral rights for the publications made accessible in the Research Explorer are retained by the authors and/or other copyright owners and it is a condition of accessing publications that users recognise and abide by the legal requirements associated with these rights.

\section{Takedown policy}

If you believe that this document breaches copyright please refer to the University of Manchester's Takedown Procedures [http://man.ac.uk/04Y6Bo] or contact uml.scholarlycommunications@manchester.ac.uk providing relevant details, so we can investigate your claim.

\section{OPEN ACCESS}




\title{
Integrated Make-or-Buy and Facility Layout Problem with Multiple Products
}

\author{
Hillebrand, Bernd ${ }^{*}++/$ Pishchulov, Grigory * \\ * Juniorprofessur Supply Chain Management, Technische Universität Dortmund, Martin- \\ Schmeißer-Weg 12, 44227 Dortmund, Germany, bernd.hillebrand@tu-dortmund.de \\ ${ }^{+}$Lehrstuhl für Produktion und Logistik, Technische Universität Dortmund, Martin- \\ Schmeißer-Weg 12, 44227 Dortmund, Germany
}

\begin{abstract}
Layout decisions represent an important step in production planning as they significantly affect the efficiency of a facility. They typically follow after make-or-buy decisions and capacity planning of a firm, and belong to the lowest level in the hierarchy of tactical production decisions. Sequential decision making neglects however the potential interaction between consecutive planning levels and may lead to inefficiencies. We propose an approach to a simultaneous makeor-buy decision making and facility layout planning in the presence of multiple products. To this end, we introduce a generalization of the classical quadratic assignment problem that takes into account (i) the costs of outsourcing, and (ii) the impact of layout decisions on the production costs of individual products. We further propose a solution approach based on the linearization of the resulting model and derive basic properties of the optimal planning decisions.
\end{abstract}

\section{Introduction}

The tactical production planning can be divided into three different levels: The first level involves decisions about the production range, specifically about which products from the product line of the firm should be made in-house, and which should be sourced from external suppliers. The second level determines the production process and the necessary resources. The third level aims to locate resources within the firm's premises while seeking to minimize unproductive movement of material and employees in the production process - with the general objective of cutting throughput times and saving costs (cf. Tompkins et al., 2010). A production site of the firm is typically referred to as facility (Reid and Sanders, 2007). In general, the decision about the assignment of different objects to loca- 
tions within a facility is called a facility layout problem (FLP) (cf. Drira et al., 2007).

The process of tactical production planning is organized hierarchically and therefore may neglect certain interactions between the different planning steps. For example, including an additional product into the production range typically increases the material flow within the production process. The additional material flow costs must not necessarily be attributed to the new product exclusively - as they may be determined to a certain extent by the existing facility layout, which has been tailored to accommodate the existing material flow. Attempting to minimize the material flow costs associated with each individual product is however likely to lead to conflicting choices with regard to the facility layout. Therefore, re-designing the existing layout to accommodate the entire production range would require seeking a trade-off between the material flow costs related with all of the products. The material flow costs of the resulting optimal layout that go beyond the total of the material flow costs which would have been generated by the production of each individual product in isolation from the others on the respective optimal layout, can be defined as complexity costs of facility layout (Gössinger, 2008).

In the present work we propose an approach to a simultaneous make-or-buy decision making and facility layout planning in the presence of multiple products. It simultaneously takes into account (i) the costs of product outsourcing, and (ii) the impact of facility layout on the production costs of individual products, leading to a managerial decision which is jointly optimal from both perspectives: choosing a production range and a facility layout. To focus on the main effects of such integrated decision making, we assume that the make-or-buy decision has no influence on the process and resource planning, so that the second decision level of the tactical production planning shall not be part of our analysis. However, in Section 6 we explain how this decision level can be integrated into the proposed decision-making approach. The rest of the paper is structured as follows. In Section 2 we briefly review the relevant literature. Section 3 describes the problem under study and the modeling approach. In Section 4 we study basic properties of the optimal planning decisions and illustrate them on a numerical example. Section 5 presents a solution approach based on the linearization of the underlying optimization problem. In Section 6 we conclude with a summary and an outlook.

\section{Literature review}

Facility layout planning comprises a broad range of planning tasks (cf. Tompkins et al., 2010) of which we restrict our attention in the present work to those of them that are represented by the FLP. The Quadratic Assignment Problem (QAP) of Koopmans and Beckmann (1957) can be considered as one of the earliest models which suits several common variants of facility layout problems. The QAP is an 
integer quadratic optimization problem, which models the task of optimally assigning a number of interconnected objects to a number of given locations by minimizing the distance-weighted connection intensity (manifested e.g. in the resulting material flow) between the objects.

Subsequent research introduced several additional aspects to this problem setting, such as specific layout configurations and special handling devices (e.g. Afentakis, 1989, Kouvelis and Chiang, 1992; Braglia, 1996), and demonstrated a range of applications of the QAP in diverse facility layout planning contexts, e.g. in universities (Dickey and Hopkins, 1972), hospitals (Elshafei, 1977) and forest zoning (Bos, 1993). The QAP is considered to be one of the most popular models of the FLP (Loila et al., 2007).

The QAP is often solved by its reduction to an (mixed-)integer linear program ((M)ILP). The most often cited reason for this is to obtain better interim solutions within problem-specific solution procedures to increase their efficiency or to form the basis for new solution approaches. The linearization of Lawler (1963) is one of the first approaches to obtaining lower bounds for this purpose. Kaufman and Broeckx (1978) provide another linearization for obtaining lower bounds when applying Benders' decomposition (Benders, 1962). This linearization was improved by Xia and Yuan (2006) as well as Zhang et al. (2013). Also Bazaraa and Sherali (1980) use their linearization with Benders' decomposition. Christofides et al. (1980) provide a linearization to reduce complexity in the solution process of large-scale instances by means of a decomposition approach. Frieze and Yadegar (1983) apply a Lagrange relaxation to their linearization in order to tighten the bounds. Adams and Johnson (1994) provide another linearization to further improve the bounds. Helber et al. (2014) apply a fix-and-optimize heuristic (Helber and Sahling, 2010) to their linearization of the QAP.

In comparison to the different variants of FLPs, where the intensity of the material flow between the objects is pre-defined, only a few contributions mention variable material flow between the objects. A multi-period setting was investigated for the first time by Rosenblatt (1986). Such problem settings are called dynamic facility layout problems, as the material flow is specified for each individual period. A fuzzy representation of a variable material flow (e.g. Grobelny, 1987; Dweiri and Meier, 1996; Enea et al., 2005) and a scenario-based robustness analysis (Rosenblatt and Lee, 1987) are examples of including a restricted knowledge about the material flow intensity into the facility layout planning. Still, the decision maker cannot influence the actual realization of the flow intensity in these problem settings.

Note that the decision maker can however influence the flow intensity by varying the production range - e.g., by outsourcing the production of certain products (see e.g. Gunasekaran et al., 2015, for a comprehensive discussion of outsourcing decision making). In order to evaluate such opportunity, the material flow needs to be considered at the product level. We are aware of an only study 
that adopts this perspective in the context of facility layout planning - that by Bazaraa and Sherali (1980), based on the earlier work by Graves and Whinston (1970). However in their problem setting, the production range is not subject to change. To our best knowledge, the present work is the first one to address makeor-buy decisions and facility layout planning within an integrated approach.

For a more profound insight to the different problems setting in FLPs please see Singh and Sharma (2006) as well as Drira et al. (2007).

\section{The integrated make-or-buy and facility layout problem}

\subsection{Problem description and modeling assumptions}

Consider a company which has to satisfy the given demand for a set $P$ of products within a specific period. The length of this period corresponds usually to the range of tactical production planning which depends on the industry and spans typically a number of years. The entire demand for each single product can either be produced in-house or purchased from an outside supplier. As in most of FLPs studied in the literature, we assume that the demand is deterministically known (cf. Drira et al., 2007). The respective make-or-buy decision with regard to product $p \in P$ is represented by the variable $q_{p} \in\{0 ; 1\}$. To make the products inhouse, it is necessary to arrange a set $I$ of objects (e.g. equipment units or machines) on the set $K$ of locations within the facility. For brevity, we will refer to the objects as machines. Every machine can be placed on any location; one location can accommodate at most one machine. For this reason, $|I| \leq|K|$ is required.

The decision about the assignment of machine $i$ to location $k$ is represented by the variable $u_{i k} \in\{0 ; 1\}$. Determining their values for all $i, k$ defines therefore the layout of the facility. We assume that set $I$ of machines to be located is not affected by the make-or-buy decision. As an example, consider a joinery that ships furniture products to a retail chain.

If $q_{p}=1$, the direct material flow from machine $i$ to machine $j$ causes costs $\lambda_{p i j}$ per distance unit, where $\lambda_{p i j} \geq 0$ for every $i, j \in I$ is determined by the production process of product $p$. The distance between the locations $k$ and $l$ is represented by $d_{k l}$. If a specific product $p$ is not made in-house $\left(q_{p}=0\right)$, the entire demand for this product has to be met via outsourcing at the cost $c_{p}$. In general, $c_{p}$ should represent the total cost of procuring the entire demand volume of product $p$ from an external supplier, less any savings realized due to not producing product $p$ in-house - so that $c_{p}$ can also be negative. To simplify the exposition, we further assume that the production costs of any product, except for that part of 
theirs which results from the material flow within the facility layout, remain unaffected by the make-or-buy decision.

It should be noted that the total procurement costs are determined in the industry practice by a broad range of factors, both financial and non-financial, e.g. the purchase price, supplier service level, monitoring costs, and alliances' risk perspectives, to name a few (see Gunasekaran et al., 2015). For the purposes of the present study we assume that the outsourcing cost $c_{p}, p \in P$, accounts in our model for all relevant procurement cost factors. Alternatively, it can be interpreted as a penalty cost for not producing the respective product at all.

The objective is to minimize the total costs of sourcing by choosing between production and outsourcing for each product $p$, as represented by variables $q_{p}, p \in P$, and a choosing a facility layout represented by variables $u_{i k}, i \in I, k \in K$. This defines the integrated make-or-buy and facility layout problem.

\subsection{Model statement}

The following optimiziation problem seeks to determine the optimal solution of the integrated make-or-buy and facility layout problem:

$$
\begin{aligned}
& T C^{*}:=\min \sum_{p \in P} \sum_{i \in I} \sum_{j \in I} \sum_{k \in K} \sum_{l \in K} q_{p} \cdot \lambda_{p i j} \cdot d_{k l} \cdot u_{i k} \cdot u_{j l}+\sum_{p \in P}\left(1-q_{p}\right) \cdot c_{p} \\
& \sum_{k \in K} u_{i k}=1 \quad \forall i \in I \\
& \sum_{i \in I} u_{i k} \leq 1 \quad \forall k \in K \\
& u_{i k} \in\{0,1\} \quad \forall i \in I ; k \in K \\
& q_{p} \in\{0,1\} \quad \forall p \in P
\end{aligned}
$$


Problem (1)-(5) is an extension of the QAP. The first part of (1) represents the total material flow costs of in-house production. Specifically, if machine $i$ is assigned to location $k$ and machine $j$ is assigned to location $l$, then $u_{i k}=u_{j l}=1$. Should product $p$ be made in-house $\left(q_{p}=1\right)$, the cost of material flow between machines $i$ and $j$ associated with that product must be taken with the factor $d_{k l}$, which accordingly represents the distance between the machines. The second part of (1) expresses the total costs of outsourcing the products, which are not produced in-house. The total of material flow costs and outsourcing costs has to be minimized. Constraints (2) and (3) express that each machine is assigned to exactly one location and that at most one machine is assigned to each location. Constraints (4) and (5) define the feasible range of decision variables. Note that (1)-(5) is a non-convex integer optimization problem with a cubic objective function, $|I| \cdot|K|+|P|$ binary variables and $|I|+|K|$ linear constraints. ${ }^{1}$

In the next section we derive basic properties of optimal solutions of problem (1)-(5) and illustrate them by a numerical example.

\section{Properties of optimal make-or-buy and facility layout decisions}

\subsection{Superadditivity of minimum flow costs}

In this section we establish a basic property of material flow costs induced by a subset of products in their respectively optimal facility layout: This property states that merging two subsets of products for in-house production cannot reduce the material flow costs - compared to the total of these costs which would have arisen if each subset of products would have been produced in isolation on the respectively optimal layout. This property can be described as superadditivity of the material flow costs in the respectively optimal layout as a function of the set of products made in-house. Let $S \subseteq P$ be a non-empty set of products and $\Lambda_{S}$ the optimization problem (1)-(4) with $q_{p}=1$ for every $p \in S$ and $q_{p}=0$ as well as $c_{p}=0$ for every $p \in P \backslash S$. Consequently, the value of $q_{p}$ for $p \in P$ is not vari-

\footnotetext{
1 Note that integrality of make-or-buy decision variables expressed by constraint (5) can be relaxed, meaning essentially that any product demand can be shared between in-house production and outsourcing. This would render the problem under study to an integrated make-and-buy and facility layout problem. It can however be shown that such relaxation does not improve the optimal objective value in (1). We omit the detailed proof here for reasons of space.
} 
able anymore and represents a parameter of problem $\Lambda_{S}$. Further, let $Z_{S}^{*}$ be the optimal objective function value of $\Lambda_{S}$ and $L_{S}^{*}=\left\{u_{i k}^{*} \mid i \in I, k \in K\right\}$ its optimal solution. Thus $Z_{S}^{*}$ displays the minimal material flow costs resulting from in-house production of product set $S$. There holds the following

Proposition 1. $Z_{S}^{*}+Z_{P \backslash S}^{*} \leq Z_{P}^{*} \quad \forall S \subset P, S \neq \varnothing$.

Proof: It is easy to see that the objective function of $\Lambda_{P}$ is the sum of the objective functions of problems $\Lambda_{S}$ and $\Lambda_{P \backslash S}$. Note further that all three problems $\Lambda_{P}$, $\Lambda_{S}$ and $\Lambda_{P \backslash S}$ have an identical feasible region. Furthermore, $L_{P}^{*}$ cannot deliver an objective value to problem $\Lambda_{S}$ lower than $Z_{S}^{*}$ as well as cannot it deliver an objective value to $\Lambda_{P \backslash S}$ lower than $Z_{P \backslash S}^{*}$. This leads to the assertion of the proposition.

This property implies that, compared to the exclusive in-house production of the entire set $P$, it can be more economically to restrict the set of products made inhouse to $S$ in order to achieve savings in material flow costs of the magnitude $K=Z_{P}^{*}-\left(Z_{S}^{*}+Z_{P \backslash S}^{*}\right)+Z_{P \backslash S}^{*}$. However this will lead to outsourcing costs of $\sum_{p \in P \backslash S} c_{p}$. In the case of $|P|=2$ the amount $Z_{P}^{*}-\left(Z_{S}^{*}+Z_{P \backslash S}^{*}\right)$ represents the complexity costs as defined in Section 1.

The above exposition makes clear that optimal make-or-buy decisions depend on the trade-off between complexity and material movement costs on the one hand and outsourcing costs on the other. The following section analyses accordingly the structure of optimal make-or-buy decisions.

\subsection{Structure of optimal make-or-buy decisions}

Below we investigate the dependence of optimal make-or-buy-decisions on the costs of outsourcing. We will describe this dependence as optimal make-or-buy decision policy. To analyze its structural properties we will focus on the case $P=\{1,2\}$ and vary the outsourcing costs $c_{1}$ and $c_{2}$ on the interval $[0 ; \infty)$ respectively. Let further the notation of Section 5.1 hold. For the sake of brevity, let $Z_{p}^{*} \equiv Z_{\{p\}}^{*}$ for $p \in P$. There holds the following

Proposition 2. Depending on the outsourcing costs $c_{1}$ and $c_{2}$, it is optimal to decide between in-house production and outsourcing as follows:

a) $c_{1} \leq Z_{1}^{*} \wedge c_{2} \leq Z_{2}^{*} \Rightarrow\left(q_{1}^{*}, q_{2}^{*}\right)=(0,0)$;

b) $c_{1} \geq Z_{1}^{*} \wedge c_{2} \leq \min \left\{c_{1}+Z_{2}^{*}-Z_{1}^{*} ; Z_{P}^{*}-Z_{1}^{*}\right\} \Rightarrow\left(q_{1}^{*}, q_{2}^{*}\right)=(1,0)$; 
c) $c_{1} \leq \min \left\{c_{2}+Z_{1}^{*}-Z_{2}^{*} ; Z_{P}^{*}-Z_{2}^{*}\right\} \wedge c_{2} \geq Z_{2}^{*} \Rightarrow\left(q_{1}^{*}, q_{2}^{*}\right)=(0,1)$;

d) $c_{1} \geq Z_{P}^{*}-Z_{2}^{*} \wedge c_{2} \geq Z_{P}^{*}-Z_{1}^{*} \Rightarrow\left(q_{1}^{*}, q_{2}^{*}\right)=(1,1)$.

Proof: If the condition of case a) holds then outsourcing of each product is cheaper than its exclusive in-house manufacturing on the respectively optimal layout. Note that there are four possible make-or-buy decisions: $\left\{\left(q_{1}, q_{2}\right) \mid q_{1}, q_{2} \in\{0,1\}\right\}$. Solving the problem (1)-(5) while fixing $q_{1}, q_{2}$ to particular values will result in four problem instances. A direct comparison of their objective values implies that $\left(q_{1}, q_{2}\right)=(0,0)$ results in the lowest objective value. This proves case a). The proof of the subsequent cases can be carried out by a similar approach.

The proof of Proposition 2 yields the following result:

Corollary 1. The optimal objective value of problem (1)-(5) in cases $a$ )-d) of Proposition 2 are respectively $c_{1}+c_{2}, Z_{1}^{*}+c_{2}, c_{1}+Z_{2}^{*}$ and $Z_{P}^{*}$.

Optimal make-or-buy decisions arising in cases a)-d) for the given combinations of outsourcing $\operatorname{costs} c_{1}$ and $c_{2}$ comprise four respective areas on the coordinate plane $O c_{1} c_{2}$ which are represented by areas $\mathrm{A}-\mathrm{D}$ in the illustration provided on the right of Figure 4.1.

As the figure illustrates, case a) applies when neither the exclusive in-house production of product 1 , nor that of product 2 is cheaper than outsourcing the respective product. Therefore both products will be purchased from the external supplier, which results in costs $c_{1}+c_{2}$. If $c_{1} \geq Z_{1}^{*}$, case b) applies. In this case, outsourcing is more expensive than exclusive in-house production of product 1 . Therefore product 1 will be produced and product 2 will be purchased if $c_{2}+Z_{1}^{*} \leq c_{1}+Z_{2}^{*}-$ i.e., it is not cheaper to do the opposite and, further, $c_{2} \leq Z_{p}^{*}-Z_{1}^{*}-$ i.e., the outsourcing costs of product 2 are not higher than the additional production costs; the latter include the complexity costs if they emerge. Case c) can be interpreted in a similar way. If $c_{1} \geq Z_{p}^{*}-Z_{2}^{*}$ and $c_{2} \geq Z_{p}^{*}-Z_{1}^{*}$ then case d) applies. In this case, production of both products on a shared optimal layout is cheaper than outsourcing any of the products - despite of the existence of possible complexity costs. So both products will be produced at the cost $Z_{P}^{*}$.

\subsection{Numerical example}

In the following example we consider a facility with six locations arranged in a row. Between two adjacent locations, the distance amounts to one distance unit. If there are $n$ locations between any two given locations, then the distance between the given two amounts to $n+1$ distance units. Six machines have to be located. The product set is given by $P=\{1,2\}$. Product 1 passes the machines in the fol- 
lowing order: 1-2-3-4-5-6, for the second product this order is respectively 5-2-3$4-1-6$. The direct transfer of a product from one machine to another results in material flow costs of one currency unit per distance unit.
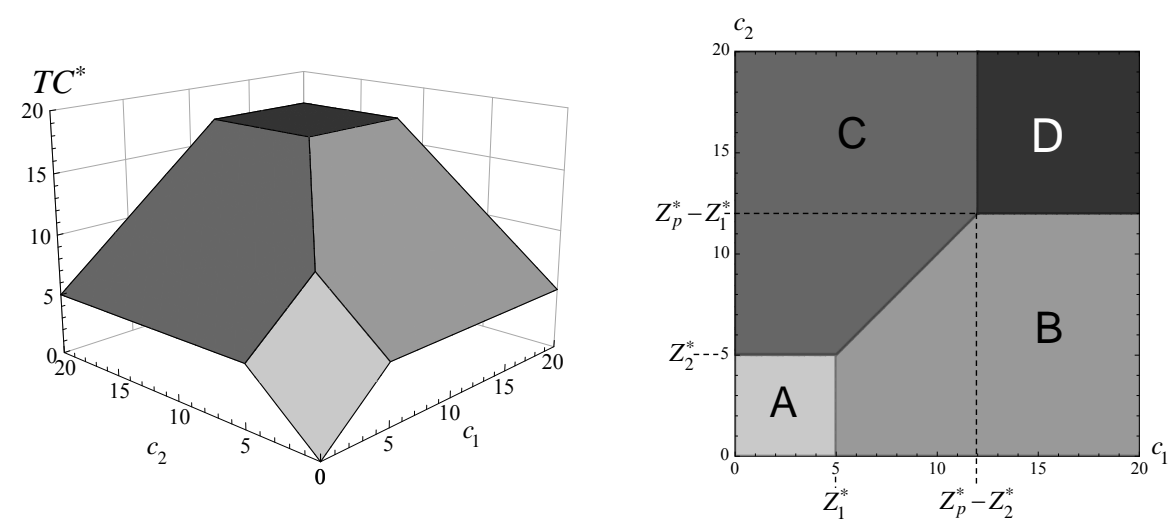

Figure 4.1: Optimal make-or-buy decisions and resulting objective values as a function of $c_{1}, c_{2}$

Firstly we compute the objective values $Z_{1}^{*}, Z_{2}^{*}$ and $Z_{P}^{*}$ using CPLEX v. 12.5 ; these have been found to be 5,5 and 17 respectively. We then let the outsourcing costs $c_{1}$ and $c_{2}$ vary in the range from 0 to 20 and determine the optimal make-or-buy decisions by means of Proposition 2. The right-hand part of Figure 4.1 illustrates cases a)-d) from Proposition 2. Its left-hand part shows the respective optimal objective values of problem (1)-(5) as per Corollary 1. The four colored faces of the graph correspond to one of cases a)-d). It can be seen that the projection of the graph on the horizontal coordinate plane yields the right-hand part of Figure 4.1.

If a layout exists, which is optimal for both products in exclusive in-house production, then the boundary between areas $\mathrm{B}$ and $\mathrm{C}$ collapses to a point; there are no complexity costs in this case.

Note that other FLPs investigated in the literature are not equipped with the possibility to outsource products. But as shown on the left-hand part of Figure 4.1, it might be an economically attractive option because of the possible presence of complexity costs.

It may be impractical or impossible to derive optimal make-or-buy and facility layout decisions for larger instances of problem (1)-(5) using a general-purpose solver as it represents an integer non-convex optimization problem. An established solution approach which guarantees an exact solution or at least a guaranteed quality of approximate solutions consists in the reduction of the problem at hand to a mixed integer linear program and solving it with respective state-of-the-art methods. This approach will be pursued in the following section. 


\section{Solution approach via linearization}

On the first sight, it appears logical to use one of the existing linearizations of the QAP to solve problem (1)-(5) by transforming it to a MILP. A closer examination however reveals that the available linearizations cannot serve this purpose, or do so only to a limited extent.

With regard to the additional variables and constraints, the linearization of Kaufmann and Broeckx (1978) is known as the most compact, thus as the most efficient formulation. However adopting their linearization technique for the purpose of linearizing problem (1)-(5) would lead to quadratic constraints in the resulting optimization problem. Their linearization is further only suitable for QAPs with a symmetric distance matrix, what cannot be assumed for facilities with special handling devices, e.g. a directed loop layout (Drira et al., 2007). The other known linearization approaches will lead to a quadratic objective function with a relatively high number of additional variables and constraints.

For the above reasons, we propose a new linearization approach which reduces (1)-(5) from a problem with a cubic objective function to the following mixed-integer linear program:

$$
\begin{aligned}
& T C^{*}:=\min \sum_{k \in K} \sum_{l \in K} d_{k l} \cdot v_{k l}+\sum_{p \in P}\left(1-q_{p}\right) \cdot c_{p} \\
& v_{k l} \geq \sum_{p \in P} q_{p} \cdot \lambda_{p i j}-M_{i j} \cdot\left(2-u_{i k}-u_{j l}\right) \quad \forall i, j \in I ; k, l \in K \\
& v_{k l} \geq 0 \quad \forall k, l \in K
\end{aligned}
$$

and (2), (3), (4) \& (5)

The new decision variables $v_{k l}$ represent in the optimal solution the resulting material flow between the locations $k, l$ in currency units per distance unit. To determine the total material flow costs in the facility, they become multiplied in objective function (6) with the distance $d_{k l}$ between the respective locations.

If machines $i, j$ are placed on locations $k, l$ respectively, then the parenthetical expression in (7) turns to zero. Accordingly, constraint (7) implies $v_{k l} \geq \sum_{p \in P} q_{p} \cdot \lambda_{p i j} \cdot u_{i k} \cdot u_{j l}$ for these $i, j$. Therefore, $v_{k l}$ has to be at least as large 
as the resulting material flow between the machines in question. For all other combinations of $i, j$, a sufficiently large number $M_{i j}$ just assures that $v_{k l}$ is bounded from below by a non-positive value. For a tight linearization, we suggest to choose $M_{i j}=\sum_{p \in P} \lambda_{p i j}$. Further, constraint (8) assures the non-negativity of $v_{k l}$ in every case. Because the objective function (6) is to be minimized, $v_{k l}$ will take on the smallest possible value for every $k, l$, thus representing the material flow between $k$ and $l$. As a result, problem (2)-(8) involves $|I|^{2} \cdot|K|^{2}$ additional constraints and $|K|^{2}$ continuous variables in comparison to problem (1)-(5).

Note that the above linearization approach is not restricted to any specific product range $P$. Note further that it can also be applied to the classical QAP by setting $q_{p}=1$ for each $p \in P$.

\section{Conclusion and outlook}

The present study proposes an approach to an integrated make-or-buy and facility layout planning which takes into account interdependencies between these planning levels. We formulate a non-linear integer programming problem which underlies the suggested planning approach. Basic properties of its optimal solutions are investigated and illustrated on a numerical example. Furthermore, we propose a linearization of the said problem to facilitate its solution with conventional tools. In addition to the above, the present study provides an operationalization of the notion of complexity costs in the context of facility layout decisions.

It should be noted that the second level of tactical production planning namely that of production process and resource planning - can be integrated into the proposed planning approach by taking into account the decision with regard to the acquisition of machines comprising set $I$. This is achieved by incorporating the following constraint into problem (1)-(5) as well as (2)-(8):

$$
\sum_{p \in P}\left[q_{p} \cdot \sum_{j \in J}\left(\lambda_{p i j}+\lambda_{p j i}\right)\right] \leq \sum_{j \in J}\left(M_{i j}+M_{j i}\right) \cdot \sum_{k \in K} u_{i k} \quad \forall i \in I
$$

Furthermore, the expression of the objective function in (1) as well as (6) has to involve the additional term $\sum_{i \in I} \sum_{k \in K} a_{i k} \cdot u_{i k}$, where $a_{i k}$ is the cost of ownership of machine $i$ when it is employed at location $k$, and constraint (2) has to be changed to an inequality with the less-or-equal sign.

Future research should attend this model extension, as well as focus on deriving solution properties for a larger number of products and gaining insights into 
the magnitude of complexity costs by conducting a numerical study in a variety of settings. Furthermore, it would be of interest to address the following problem settings in the future research: Division of labor between multiple facilities of the company, planning over product life-cycles, as well as problem settings with supply- or demand-side uncertainties. Last but not least, it is essential to develop efficient heuristic solution methods for the problem under study.

\section{Acknowledgments}

We are thankful to Rolf Wendt of TU Dortmund University for the useful insights with regard to relaxation of the problem referred to in footnote 1.

\section{References}

Adams and Johnson (1994) Adams, W. P. and T. A. Johnson, 1994: Improved linear programming-based lower bounds for the quadratic assignment problem. Quadratic Assignment and Related Problems, P. M. Pardalos and H. Wolkowicz, eds., DIMACS Series in Discrete Mathematics and Theoretical Computer Science, 16, 43-75.

Afentakis (1989) Afentakis, P. A., 1989: Loop layout design problem for flexible manufacturing systems. International Journal of Flexible Manufacturing Systems, 1, 143-175.

Bazaara and Sherali (1980) Bazaraa, M. S. and H. D. Sherali, 1980: Benders' partitioning scheme applied to a new formulation of the quadratic assignment problem. Naval Research Logistics Quarterly, 27, 29-41.

Benders (1962) Benders, J. F., 1962: Partitioning procedures for solving mixed-variables programming problems. Numerische Mathematik, 4, 238-252.

Bos (1993) Bos, J., 1993: Zoning in Forest Management: A quadratic assignment problem solved by simulated annealing. Journal of Environmental Management, 37, 127-145.

Braglia (1996) Braglia, M., 1996: Optimization of a simulated-annealing-based heuristic for single row machine layout problem by genetic algorithm. International Transactions in Operational Research, 3, 37-49.

Christofides et al. (1980) Christofides, N., A. Mingozzi and P. Toth, 1980: Contributions to the quadratic assignment problem. European Journal of Operations Research, 4, 243-247.

Dickey and Hopkins (1972) Dickey, J. W. and J. W. Hopkins, 1972. Campus building arrangement using topaz. Transportation Research, 6, 59-68.

Drira et al. (2007) Drira, A., H. Pierreval and S. Hajiri-Gabouj, 2007: Facility layout problems: A survey. Annual Reviews in Control, 31, 255-267.

Dweiri and Meier (1996) Dweiri, F. and F. A. Meier, 1996: Application of fuzzy decisionmaking in facilities layout planning. International Journal of Production Research, 34, $3207-$ 3225 .

Elshafei (1977) Elshafei, A. N., 1977: Hospital layout as a quadratic assignment problem. Operations Research Quarterly, 28, 167-179.

Enea et al. (2005) Enea, M., G. Galante and E. Panascia, 2005: The facility layout problem approached using a fuzzy model and a genetic search. Journal of Intelligent Manufacturing, 16, 303-316.

Frieze and Yadegar (1981) Frieze, A. M. and J. Yadegar, 1981: An algorithm for solving 3dimensional assignment problems with applications to scheduling a teaching practice. Operations Research, 32, 989-995.

Gössinger (2008) Gössinger, R., 2008: Produktion und Logistik. In: Corsten, H. and M. Reiß, 2008: Betriebswirtschaftslehre, $4^{\text {th }}$ ed., Vol. 1, Chapter E, 443-539. 
Gunasekaran et al. (2015) Gunasekaran, A., Z. Irani, K.-L. Choy, L. Filippi, T. Papadopoulos, 2015: Performance measures and metrics in outsourcing decisions: A review for research and applications. International Journal of Production Economics, 161, 153-166.

Graves and Whinston (1970) Graves, G. W. and A. B. Whinston, 1970: An algorithm for the quadratic assignment problem. Management Science, 17, 453-471.

Grobelny (1987) Grobelny, J., 1987: The fuzzy approach to facility layout problems. Fuzzy Sets and Systems, 23, 175-190.

Helber and Sahling (2010) Helber, S. and F. Sahling 2010. A fix-and-optimize approach for the multi-level capacitated lot sizing problem. International Journal of Production Economics, $123,247-256$.

Helber et al. (2014) Helber, S., D. Böhme, F. Oucherif, S. Lagershausen and S. Kasper, 2014: A hierarchical facility layout planning approach for large and complex hospitals. Diskus sionspapiere - Hannover Economic Papers, 527, 1-27.

Kaufman and Broeckx (1978) Kaufman, L. and F. Broeckx, 1978: An algorithm for the quadratic assignment problem using Bender's decomposition. European Journal of Operation Research, 2, 204-211.

Koopmans and Beckmann (1957) Koopmans, T. C. and M. Beckmann, 1957: Assignment Problems and the Location of Economic Activities. Econometrica, 25, 53-76.

Kouvelis and Chiang (1992) Kouvelis, P., and W. C. Chiang, 1992: A simulated annealing procedure for the single row layout problems in flexible manufacturing systems. International Journal of Production Research, 30, 717-732.

Lawler (1963) Lawler, E. L., 1963: The Quadratic Assignment Problem. Management Science, 9, 586-599.

Loila et al. (2007) Loila, E. M., N. M. M. de Abreu, P. O. Boaventura-Netto, P. Hahn, and T. Querido, 2007: A survey for the quadratic assignment problem. European Journal of $O p$ erational Research, 176, 657-690.

Reid and Sanders (2007) Reid, R. D. and N. R. Sanders, 2007: Operations Management, $3^{\text {rd }}$ ed., Hoboken, NJ: Wiley.

Rosenblatt (1986) Rosenblatt, M. J., 1986: The dynamics of plant layout. Management Science, $32,76-86$.

Rosenblatt and Lee (1987) Rosenblatt, M. J. and H. L. Lee, 1987: A robustness approach to facilities design. International Journal of Production Research, 25, 479-486.

Singh and Sharma (2006) Singh, S. P. and R. R. K. Sharma, 2006: A review of different approaches to the facility layout problems. International Journal of Advanced Manufacturing Technology, 30, 425-433.

Tompkins et al. (2010) Tompkins, J. A., J. A White, Y. A. Bozer and M. A. Tanchoco, 2010: Facilities Planning, $4^{\text {th }}$ ed., Hoboken, NJ: Wiley.

Xin and Yuan (2006) Xia, Y. and Y.-X. Yuan, 2006: A new linearization method for quadratic assignment problems. Optimization Methods and Software, 21, 803-816.

Zhang et al. (2013) Zhang, H., C. Beltran-Royo and L. Ma, 2013: Solving the quadratic assignment problem by means of general purpose mixed integer linear programming solvers. Annals of Operations Research, 207, 261-278. 